\title{
PROUD-based method for simple real-time in-line characterization of propagation-induced distortions in NRZ Data Signals
}

\author{
hugo F. Martins, ${ }^{1,}{ }^{*}$ Juan Pastor-Graells, ${ }^{2}$ Luis Romero Cortés, ${ }^{3}$ Daniel \\ Piote, ${ }^{1}$ Sonia Martin-Lopez, ${ }^{2}$ José Azaña, ${ }^{3}$ Miguel Gonzalez-Herraez, ${ }^{2}$
}

${ }^{1}$ FOCUS S.L., C/ Orellana, 1, 1ㅇ Izqda, 28004 Madrid, Spain

${ }^{2}$ Departamento de Electrónica, Universidad de Alcalá, Escuela Politécnica Superior, 28871 Madrid, Spain

IInstitut National de la Recherche Scientifique - Energie, Matériaux et Télécommunications (INRS-EMT), 1650, Boul. Lionel-Boulet, Varennes, Québec, Canada J3X 1S2

*Corresponding author: hugo.martins@focustech.eu

Received XX Month XXXX; revised XX Month, XXXX; accepted XX Month XXXX; posted XX Month XXXX (Doc. ID XXXXX); published XX Month XXXX

A simple, in-line method for real-time full characterization (amplitude and phase) of propagation distortions arising due to group velocity dispersion and self-phase modulation on 10-20 Gbps transmitted NRZ optical signals is reported. It is based on phase reconstruction using optical ultrafast differentiation (PROUD), a linear and self-referenced technique. The flexibility of the technique is demonstrated by characterizing different data stream scenarios. Experimental results were modelled using conventional propagation equations, showing good agreement with the measured data. It is envisaged that the proposed method could be used in combination with DSP techniques for the estimation and compensation of propagation distortions in fiber links, not only in conventional IM/DD systems but also in coherent systems with advanced modulation formats. (C) 2015 Optical Society of America

OCIS codes: (060.1155) All-optical networks; (060.1660) Coherent communications; (060.5060) Phase modulation; (060.5530) Pulse propagation and temporal solitons; (190.3270) Kerr effect; (130.2035) Dispersion compensation devices;

http://dx.doi.org/10.1364/OL.99.099999

With the increase of data bit rates and fiber lengths (which often reach hundreds of $\mathrm{km}$ ) in modern fiber communication systems, the management of propagation-induced distortion becomes an increasingly important issue, as it can severely limit the performance of optical communication links. If the nature of such distortion mechanisms in a given fiber link is known beforehand, a number of compensation techniques can be conceived. The compensation of Group Velocity Dispersion (GVD) and self-phase modulation (SPM) can be realized by physically propagating optical pulses through optical media with opposite GVD/nonlinear parameters [1], or by electronically designing the pulses to meet a certain desired shape [2],[3], thus achieving distortion-free pulses at the output of the link. Digital post-detection compensation of distortion is also possible in both linear and nonlinear regimes [4]. In all the mentioned cases, an accurate characterization of the complex field of the optical pulses after the propagation is required, in order to have an accurate model of the response of the link and compensate distortions accordingly.

Conventional square-law fast photodetectors can be used to accurately characterize the instantaneous power distribution of a given optical signal, but provide no information regarding its phase profile. A number of techniques that can be used to characterize arbitrary phase variations can be found in the literature [5], but these typically requirea coherent detection system (i.e., a local oscillator and a synchronization mechanism), which represent a complex technological solution that may not be always available. Well-known techniques such as Frequency-Resolved Optical Gating (FROG) [6] and Spectral Phase Interferometry for Direct Electric-Field Reconstruction (SPIDER) [7] overcome this limitation by using a self-referenced setup (i.e., one that doesn't require a local oscillator). However, these techniques are typically not ideal, as these rely in the use of nonlinearities, which can prove to be a drawback in the characterization of low-power telecommunications signals. In this context, Phase Reconstruction using Optical Ultrafast Differentiation (PROUD) is a set of direct selfreferenced techniques well adapted for the characterization of lowpower telecom signals [8]-[11]. Nonetheless, to our knowledge, no results have been reported in the literature to validate the use of PROUD for signal characterization in fiber-optics propagation experiments.

In this work, we develop and successfully validate a simple and practical balanced time-domain balanced PROUD setup that is specifically optimized for the characterization of telecom signals in a real-time fashion. The effectiveness of this in-line method is demonstrated by evaluating the impact of SPM and GVD in a 10-20 Gbps NRZ optical link. Experimental were modeled using conventional propagation equations, showing good agreement with the measured data. The chromatic dispersion and nonlinear coefficients of the fiber were easily and precisely quantified.

When propagating through a fiber, the complex field of an optical pulse will experience degradation due to several effects. For typical Cband telecom signals (around the $1.55 \mu \mathrm{m}$ wavelength), with "wellbehaved" pulse shapes and bit modulation rates well below $200 \mathrm{GHz}$ (i.e., pulse widths $>5 \mathrm{ps}$ ), the pulse propagation can be accurately 
described by solving the nonlinear Schrödinger equation for the scalar electric field envelope $A(z, t)[12]$

$$
\frac{\partial A}{\partial z}+\frac{i \beta_{2}}{2} \frac{\partial^{2} A}{\partial t^{2}}-\frac{\beta_{3}}{6} \frac{\partial^{3} A}{\partial t^{3}}+\frac{\alpha}{2} A=i \gamma|A|^{2} A
$$

where $\gamma$ is the nonlinear fiber coefficient, $\beta_{2}$ the GVD coefficient, $\beta_{3}$ the third-order dispersion coefficient and $\alpha$ is the attenuation coefficient. In this model, high order dispersion and nonlinear effects, as well as scattering processes are neglected [12]. For convenience, the reference of times is considered to travel at the group velocity of the central frequency component, and, as such, the time of flight in the fiber is disregarded in the calculations. For standard single-mode fibers (SMF), the zero of dispersion wavelength is close to $1.3 \mu \mathrm{m}$ and the effects of $\beta_{3}$ are usually negligible at $1.55 \mu \mathrm{m}$. In this case, GVD and SPM are the most important phenomena to be accounted for [12].

GVD represents the fact that the group velocity of a wave packet traveling through a dispersive medium is frequency-dependent. This introduces nonlinear phase variations along the different frequency components of the waveform (leaving the power spectral density unaltered). For a transform-limited Gaussian pulse, second-order dispersion will introduce a linear frequency chirp (i.e., a parabolic phase shift) on the pulse, with an associated pulse broadening, which can limit the data rate. In the anomalous dispersion regime $\left(\beta_{2}<0\right)$, the blue (red) shifted frequency components are moved towards the leading (trailing) edge of the pulse.

SPM, is a result of the Kerr nonlinearity of the fiber [12]. For long pulses, neglecting the effect of GVD, pure SPM leads to a nonlinear phase shift $\Phi_{N L}(\mathrm{t})$ proportional to the instantaneous power of the pulse $|A(t)|^{2}$ after propagation over a fiber of length $L$ :

$$
\phi_{N L}(z=L, t)=L_{e f f} \gamma|A(t)|^{2},
$$

where $L_{\text {eff }}=\left(1-e^{-\alpha L}\right) / \alpha$ is the effective fiber length. While pure SPM does not change the instantaneous power, the variations of $\Phi_{N L}(\mathrm{t})$ are translated into variations of the instantaneous frequency $\omega_{\text {inst }}(t)$, which leads to spectral broadening, with red (blue) frequency shifts occurring in the leading (trailing) edge of the pulse, for a Gaussian-like pulse. The combined effect of GVD and SPM will introduce undesired distortion in both the spectrum and the temporal shape of the pulse. Thus, SPM limits the peak power of the pulses.

The impact of GVD will be higher for shorter pulses (broad spectral content) while the impact of SPM will be higher for sharper pulse power variations. With anomalous dispersion $\left(\beta_{2}<0\right)$ the effects of SPM and GVD can mutually cancel under certain conditions, leading to solitonlike propagation. In any case, an accurate measurement of the instantaneous phase and frequency along the pulse profile would be important, in order to allow for an accurate characterization and compensation of the propagation-induced distortions. For this purpose, this paper demonstrates a simple in-line technique based on PROUD.

Balanced Time-domain PROUD [10] is a linear and self-referenced technique which allows for the reconstruction of the complex field (phase and amplitude) of an optical signal $x(t)$, with central frequency $\omega_{0}$. The technique uses the optical power profiles (acquired using common square-law photodetectors) of the signal, $|x(t)|^{2}$, and two signals obtained from $x(t)$ after real-time photonic differentiation: $\left|y_{+}(t)\right|^{2}$ and $\left|y_{-}(t)\right|^{2}$. Here, $y_{+}(t)$ and $y_{-}(t)$ can be obtained by inputting $x(t)$ into two linear time-invariant frequency filters with an amplitude response that is linear with the optical frequency, i.e., with spectral transfer functions $D_{ \pm}(\omega)= \pm S\left(\omega_{0}-\omega \pm \Delta \omega\right)$, with the same central frequencies as the carrier frequency of the input optical signal $x(t), \omega_{0}$, same (positive) frequency shift $\Delta \omega$ between $\omega_{0}$ and the resonance frequency (i.e., frequency at which the spectral transfer function reaches zero) of the differentiator, and opposite slopes $\pm S$. The differential output optical instantaneous power (obtained by balance photodetection of the filters outputs $y_{+}(t)$ and $\left.y_{-}(t)\right)$ can be easily demonstrated to be [10],[11]:

$$
|y(t)|^{2}=\left|y_{+}(t)\right|^{2}-\left|y_{-}(t)\right|^{2}=4 S^{2} \Delta \omega|x(t)|^{2} \omega_{\text {inst }}(t),
$$

where $\omega_{\text {inst }}=\omega-\omega_{0}$ is the instantaneous frequency shift of the signal $x(t)$ with respect to $\omega_{0}$. This allows a direct measurement of the instantaneous frequency profile of the input signal (after normalization by the reference signal $\left.|x(t)|^{2}\right)$.

The experimental setup is shown in Fig. 1a. A laser diode (LD) operating at $1547.66 \mathrm{~nm}$ followed by a $20 \mathrm{GHz}$ external optical modulator and an amplification and filtering scheme - using an erbiumdoped fiber amplifier (EDFA) followed by a narrowband tunable $10 \mathrm{GHz}$ optical filter - were used to generate a 10-20 Gbps data stream with optical pulses of variable peak power $P_{0}$. After propagation over a standard SMF spool, the complex field of the pulses $x(t)$ was characterized using a time-domain balanced PROUD detection scheme. A polarization scrambler (PS) was used to avoid polarization dependence issues in the PROUD filter.

In the detection stage, the output of the fiber was split using a calibrated 50/50 coupler: one output was used as the PROUD reference signal $|x(t)|^{2}$, while the other output was injected into a commercial $100 \mathrm{GHz}$ Dense Wavelength Division Multiplexer (DWDM) centered at $1547,32 \mathrm{~nm}$, (manufactured by Opneti). This DWDM filter shows two complementary linear spectral responses at the wavelength of interest in the pass and reflect channel (see Fig. 1b). In particular, the pass (reflect) channel was used as positive $D_{+}$(negative $D$-) slope linear optical frequency filter with $D_{ \pm}(\omega)= \pm S\left(\omega_{0}-\omega \pm \Delta \omega\right)$, where $\omega_{0}=c /(1547.66 \mathrm{~nm})$, where $c$ is the speed of light in the vacuum, $\Delta \omega \approx 2 \pi \cdot(6 \mathrm{GHz}$ ) (corresponding to a wavelength range of $\sim 0.05 \mathrm{~nm}$ ) and $|S|=0.5 / \Delta \omega$. The two outputs of the DWDM were then used as the PROUD photonic differentiated signals $\left|y_{ \pm}(t)\right|^{2}$.

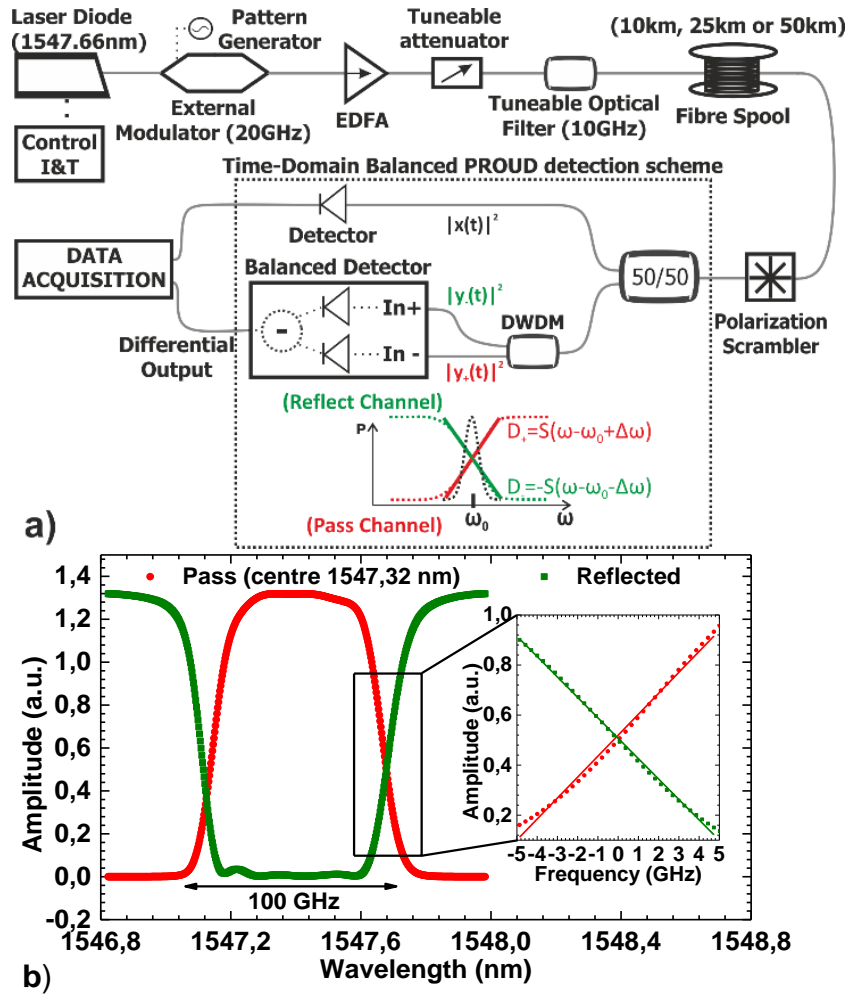

Fig. 1. a) Experimental Setup: Data signal characterization by timedomain balanced PROUD detection scheme. b) Spectral response of the DWDM used as D+/D- filter for the PROUD.

Other solutions have been tested to be used as $D_{+} / D$. filters in PROUD. Using an unbalanced Mach-Zender interferometer allows flexibility in the filter parameters, but requires a nonstandard component [9], usually with temperature stabilization [10], which increases the complexity of the system. In our case, the use of a commercial DWDM provides a stable 
and off-the-shelf low cost filter solution. For a given DWDM, the filter parametersare fixed, but with a proper choice of DWDM some flexibility in the choice of $\omega 0$ can be achieved. In our case the operation wavelength is near the ITU $100 \mathrm{GHz}$ grid channel $37(193.70 \mathrm{THz} \approx 1547.72 \mathrm{~nm})$.

The $D_{+}(D$-) signal is detected in the negative (positive) terminal of a $20 \mathrm{GHz}$ pin balanced photodetector. As a result, the output of the balanced photodetector $\left(|y(t)|^{2}=\left|y_{+}(t)\right|^{2}-|y-(t)|^{2}\right)$ is proportional to $\omega_{\text {inst }}$ (eq.3). The reference signal $|x(t)|^{2}$ was also measured using a calibrated $20 \mathrm{GHz}$ pin photodetector and a synchronization was ensured between the $|x(t)|^{2}$ and $|y(t)|^{2}$ signals. Lastly, the value of $\omega_{\text {nst }}$ is recovered by performing the normalization of the balanced signal with the reference signal (eq.3).

In order to provide a high number of sampling points and facilitate the comparison between experiments and simulations, the signals were then recorded with an 86116C sampling scope from Keysight with $80 \mathrm{GHz}$ of electrical bandwidth and an equivalent sampling of $\approx 200 \mathrm{GS} / \mathrm{s}$. In any case, real-time acquisition could also be achieved with a real-time oscilloscope, provided that the bandwidth/sampling was adjusted to the intended pulse features to be measured.

Using the experimental setup presented in Fig.1, the optical power $P(t)$, instantaneous frequency profile $v(t)=\omega_{\text {inst }}(t) / 2 \pi$ and instantaneous phase shift temporal profile $\Phi(t)$ of the data signal are directly characterized. $\Phi(t)$ is recovered using $\phi(t)=-2 \pi \int v(t) d t$, where the minus sign is due to the choice of the factor $e^{-i \omega t}$ when describing the electric wave [12]. Different distortion profiles are expected for different modulation rates or number of consecutives "1"s in a NRZ sequence, as these are equivalent to optical pulses with different width/shape. In order to show the flexibility of the technique, a variety of signals corresponding to several data sequences were characterized. In our case, only the instantaneous phase shift normalized to the input temporal phase profile (i.e., $\left.\Phi(t)=\Phi_{\mathrm{z}=\mathrm{L}}(t)-\Phi_{\mathrm{z}=0}(t)\right)$ is presented. The presence of frequency chirp in the input pulse is therefore neglected, which, in any case, was observed to be small in comparison with the chirp acquired after the propagation.

The experimental results were compared with the theoretical expectations, obtained by numerically solving eq. 1 using a split-step Fourier method with adaptive step size. The used input scalar electric field envelopes $S(z=0, t)$ were transform-limited super-Gaussian pulses:

$$
A(z=0, t)=\sqrt{P_{0}} \exp \left[-0.5(t / T 0)^{2 m}\right],
$$

where $m$ is the order of the super-Gaussian, $P_{0}$ is the peak power and $\mathrm{T}_{0}$ determines the width of the pulse. The fiber parameters were those of a standard SMF-28 at a reference wavelength of $1550 \mathrm{~nm}: \gamma=1.4 \mathrm{~W}^{-1} \mathrm{~km}^{-1}$, $\beta_{2}=15 \mathrm{ps}^{2} \mathrm{~km}^{-1}, \beta_{3}=0.1 \mathrm{ps}^{3} \mathrm{~km}^{-1}$ and $\alpha=0.2 \mathrm{~dB} \cdot \mathrm{km}^{-1}$.

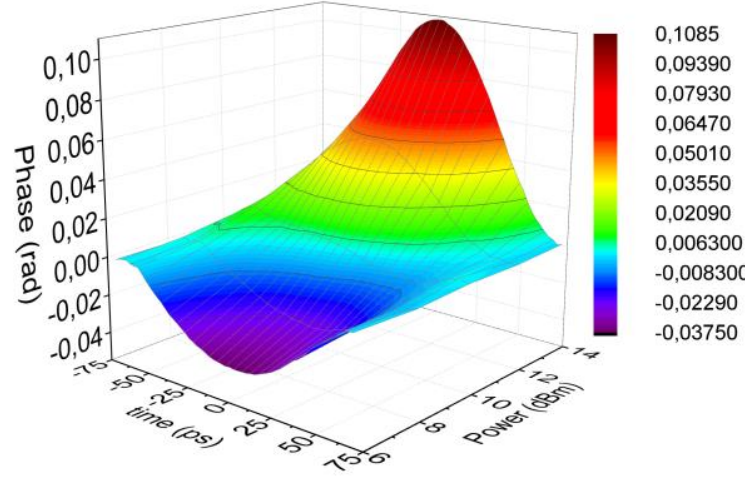

Fig. 2. Experimental characterization of $\Phi(t)$ of a single Gaussian-like pulse at 10 Gbps with $~ 90$ ps FWHM after propagation over $10 \mathrm{~km}$ of fiber, for varying input $P_{0}(6-14 \mathrm{dBm})$.

First, the $\Phi(t)$ of a single $10 \mathrm{Gbps}$ " 1 " bit after propagation over $10 \mathrm{~km}$ of fiber was characterized for varying input $P_{0}$ (6-14 dBm). A complete set of results is shown in Fig. 2. Due to the nonlinear response of the modulator, the optical pulse resembled a Gaussian optical pulse with $\sim 90$ ps full width at half maximum (FWHM). The normalized input pulse's instantaneous power profile is shown in Fig. 3c. For a Gaussian pulse under anomalous dispersion, GVD and SPM will introduce almost linear frequency chirps with opposite signs around the pulse center. The results can therefore be interpreted as follows: for $P_{0}=10 \mathrm{dBm}$, the effects of GVD and SPM are comparable and almost cancel each other. Hence, an almost flat $\Phi(t)$ is obtained. As $\mathrm{P}_{0}$ is decreased (increased) from $10 \mathrm{dBm}$, the effects of GVD (SPM) have a higher impact, thus resulting in positive (negative) and close to parabolic (Gaussian) $\Phi(t)$, as expected [12].

Fig. 3(a, b, c) and 3(d, e, f) show the $\Phi(t), v(t)$ and $P(t)$ of a single " 1 " bit (same input pulse of Fig. 2) and a sequence of five "1" bits at $10 \mathrm{Gbps}$ of a NRZ signal, respectively, for different peak power values $P_{0}$ (6-14dBm), after propagation over $50 \mathrm{~km}$ of SMF-28. For clarification, it should be noted that the leading edge of the pulses is correspondent to the negative times (i.e., signal that arrives first to the photodetector for the experimental results), and the trailing edge to the positive times of figures 2-4. With higher $\mathrm{P}_{0}$, the impact of SPM is increased: a $\Phi(t)$ approximately proportional to $P(t)$ is obtained (Fig. 3a, 3d), with red (blue) frequency shifts occurring in the leading (trailing) edge of the pulse (Fig. 3b, 3e). In the low power regime, an increase of the effect of GVD is clearly observed, with blue (red) frequency shifts occurring in the leading (trailing) edge of the pulse, where the spectral content of the pulse is broader and a gradual shift of $\Phi(t)$ towards positive phases in the center of the pulse (Fig. 3a, 3d). The impact of GVD (SPM) is observed to be higher for the shorter (longer) pulse, as expected, due to its broader (narrower) spectral content.

The measured instantaneous power profile, normalized to $P_{0}$, is given in Fig. 3 (c, f). The fiber losses are numerically compensated to facilitate pulse shape comparisons. The pulses keep its original shape, although noticeable distortions are observed, especially near the edges of the pulse in Fig. 3f. The distortions are higher for higher $P_{0}$, as expected [12].

The evolution of the pulses shown in Fig. 3 were simulated using super-Gaussian pulses as input (see eq.4) with $\mathrm{m}=1(\mathrm{a}, \mathrm{b})$ and $T_{0}=35 \mathrm{ps}$ (FWHM 80ps), $\mathrm{m}=3$ (d, e) and $T_{0}=165 \mathrm{ps}$ (FWHM $\sim 440 \mathrm{ps}$ ), showing good agreement with the experimental results.
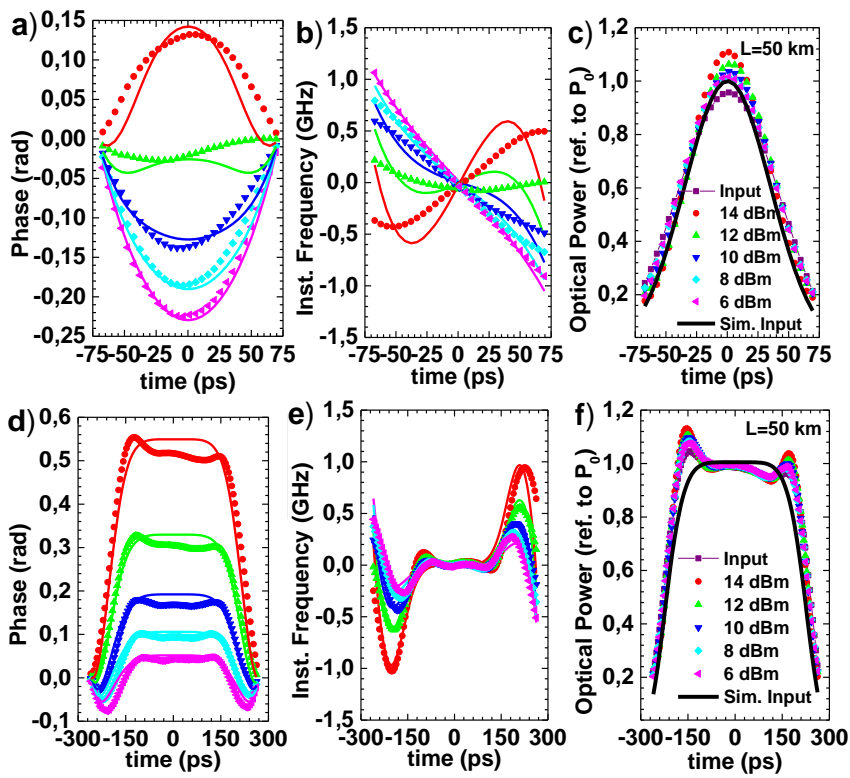

Fig. 3. Measured (dotted lines) $\Phi(t), v(t), P(t)$ for a single $\sim 90$ ps FWHM pulse (a, b, c), and five " 1 " NRZ bits corresponding to 470 ps (d, e, f) at $10 \mathrm{Gbps}$ after $50 \mathrm{~km}$ of fiber, for various values of the input $P_{0}$. Theoretical simulations (solid lines) are plotted for comparison.

Fig. 4a, 4b, 4c shows the evolution of the $\Phi(t), v(t), P(t)$ of a sequence of five consecutive " 1 " bits of a $20 \mathrm{Gbps}$ NRZ signal (an almost flat-top 
input pulse with $\sim 220$ ps FWHM) after propagation over 10, 25 and 50 $\mathrm{km}$ of fiber $\left(L_{\mathrm{eff}} \approx 8,14.8,19.5 \mathrm{~km}\right.$, with $\left.\alpha=0.2 \mathrm{~dB} \cdot \mathrm{km}^{-1}\right)$, with a relatively high $P_{0}(14 \mathrm{dBm})$. Here, the mechanisms of the propagation distortions can be clearly understood.

For the shorter fiber distances $(10,25 \mathrm{~km})$ the optical power of the pulse is high and the impact of SPM is dominant. In this case, the shape of $\Phi(t)$ (Fig. 4a) should resemble $P(t)$, with an amplitude that increases proportionally to $L_{\text {eff }}$ (see eq. 2). The correspondent $v(t)$ is also as expected (Fig. 4b): red (blue) $v(t)$ shifts occurring in the leading (trailing) edge of the pulse and a maximum $v(t)$ shift that increases with

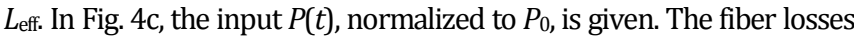
are numerically compensated in the plots, to facilitate pulse shape comparisons. It should be pointed out that the $P(t)$ of the pulse (Fig. 4c), experienced a small but noticeable deformation with the length increase, thus indicating that the effect of GVD is not negligible.

Between the distances of $25 \mathrm{~km}$ and $50 \mathrm{~km}$, the optical power decreases due to the fiber losses and the impact of SPM is reduced: a much smaller increase of the maximum $v(t)$ shift of the pulse is observed between $25 \mathrm{~km}$ and $50 \mathrm{~km}$ than between $0 \mathrm{~km}$ and $25 \mathrm{~km}$ (Fig.4b). On the other hand, the frequencies previously generated by SPM were moved closer to the pulse center, as it would be expected in the case where GVD is dominant. As for the form of $\Phi(t)$, after $50 \mathrm{~km}$ the acquired phase shift is smoothed to the point of resembling that of a Gaussian pulse (Fig.3a).

Lastly, Fig. 4d, 4e, 4f present the $\Phi(t), v(t), P(t)$ of this pulse after propagation over $\mathrm{L}=50 \mathrm{~km}$ of fiber for various values of the input $P_{0}$, presenting consistent results with those found in Fig. 3.
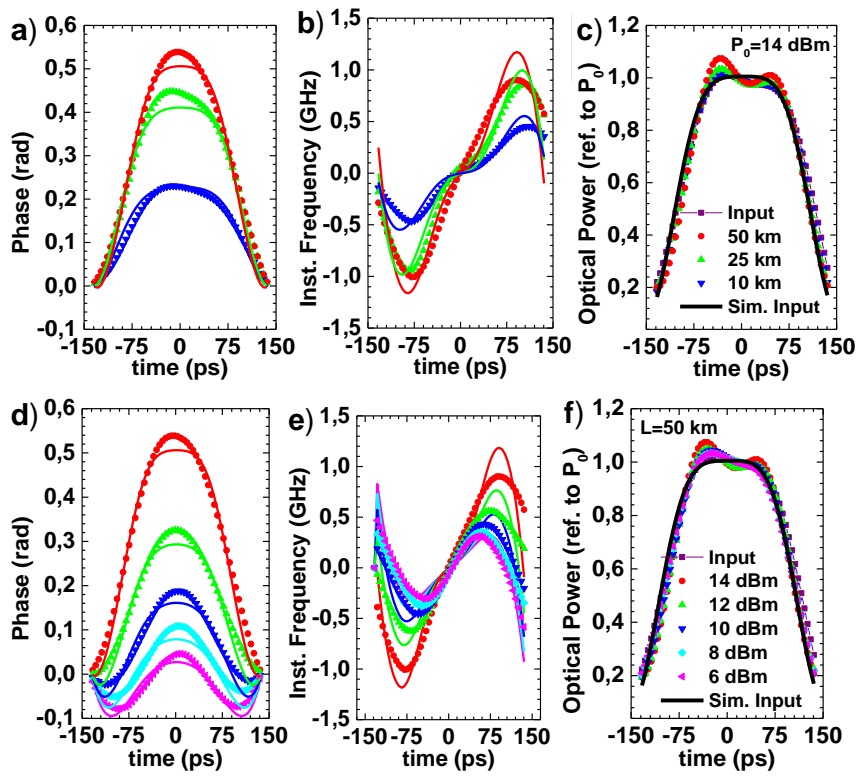

Fig. 4. Measured (dotted lines) $\Phi(t), v(t), P(t)$ for five consecutive " 1 " NRZ bits at 20 Gbps ( 220ps FWHM) with $P_{0}=14 \mathrm{dBm}$ after

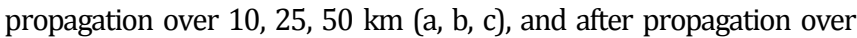
$50 \mathrm{~km}$ with $P_{0}=(6-14 \mathrm{dBm})(\mathrm{d}, \mathrm{e}, \mathrm{f})$. Theoretical simulations (solid lines) are plotted for comparison.

For comparison purposes, the presented experimental conditions were simulated using a super-Gaussian pulse (see eq. 4 ) with $\mathrm{m}=2$ and $T_{0}=82$ ps (FWHM $\approx 210 p s$ ) as input pulse, as depicted in Fig. 4c. A smoothening of the measured $v(t)$ (Fig. $4 \mathrm{~b})$ seems to occur, which could be due to the limited bandwidth of the photodetector. It should be noted that for the 10, 25, $50 \mathrm{~km}$ measurements, different fiber spools were used and, therefore, small variations in the fiber parameters are possible. The effects of GVD ( $\propto \partial^{2} A / \partial t^{2}$ ) will be much more sensitive to fluctuations in the pulse's $P(t)\left(=|A(t)|^{2}\right)$ than the effects of SPM $\left(\propto A|A|^{2}\right.$ ). In this case, higher discrepancies between the simulations and the experimental results are anticipated when the impact of GVD is higher (edges of the pulses and low input $P_{0}$ ), as it was observed. In any case, a good agreement between the theoretical simulations and experimental results is observed for all the measurements.

It should be noted that telecom signals typically use low pulse powers to avoid the impact of nonlinearities such as SPM. The pulse power range used in this work (6-14 dBm) was therefore significantly higher than those typically used in telecom signals, as it was chosen to study the limiting situation where the impact of SPM and GVD were both relevant. This allows demonstrating the flexibility of PROUD in characterizing the propagation-induced distortions in such scenario.

In conclusion, in this work, a simple, complete characterization (amplitude and phase) of optical pulses corresponding to several NRZ data signal scenarios (varying number of consecutive " 1 " bits, data rates, input peak powers and fiber propagation lengths) was demonstrated using PROUD. This linear and self-referenced technique does not require a local oscillator and its detection scheme can be assembled from standard off-the-shelf telecom components, being fully compatible with conventional optical networks. The experimental results were easily modelled using conventional propagation equations, showing good agreement with the measurements. Moreover, the fiber parameters, mainly GVD and nonlinear coefficients could be estimated from the comparison between simulations and experimental measurements, showing good agreement with those of a typical SMF. This system could potentially be used for real-time characterization of propagation distortions (not limited to SPM/GVD) induced on NRZ or other signal formats in optical communication networks and, combined with other techniques, allow for the accurate compensation of these distortions.

Funding: This work was supported by the European Research Council through Starting Grant UFINE (Grant no. 307441), the Spanish MINECO through projects TEC2012-37958-C02-01 and TEC201345265-R, and the regional programs SINFOTON-CM: S2013/MIT-2790 and EDISON (CCG2014/EXP-072). HFM acknowledges EU funding through the FP7 ITN ICONE program, gr. \#608099. JPG and SML acknowledge funding from the Spanish MINECO through a FPI and a "Ramon y Cajal" contract, respectively.

\section{References}

1. C. Paré, A. Villeneuve, and P.-A. Bélanger, Opt. Lett 21, 459 (1996).

2. R. I. Killey, P. M. Watts, V. Mikhailov, M. Glick, and P. Bayvel, IEEE Photon. Technol. Lett. 17, 714 (2005).

3. K. Roberts, C. Li, L. Strawczynski, M. O’Sullivan, and I. Hardcastle, IEEE Photon. Technol. Lett 18, 403 (2006).

4. Y. Mori, C. Zhang, K. Igarashi, K. Katoh, and K. Kikuchi, Opt. Exp. 17, 1435 (2009).

5. N. K. Fontaine, R. P. Scott, L. Zhou, F. M. Soares, J. P. Heritage, and S. J. B. Yoo, Nature Photon. 4, 248 (2010).

6. D. J. Kane, and R. Trebino, Opt. Lett. 8, 823 (1993).

7. C. laconis and I. A. Walmsley, Opt. Lett. 23, 792 (1998).

8. F. Li, Y. Park, and J. Azaña, Opt. Lett. 32, 3364 (2007).

9. H. P. Bazargani, J.-B. Quélène, P. Dumais, A. Malacarne, M. Clerici, R. Morandotti, C. L. Callender, and J. Azaña, IEEE Photon. Technol. Lett. 26, 34 (2014).

10. F. Li, Y. Park, and J. Azaña, Opt. Lett 34, 2742 (2009).

11. J. Azaña, Y. Park, and F. Li, Opt. Commun. 284, 3772 (2011).

12. G. P. Agrawal, Nonlinear Fiber Optics, 3rd ed., Academic (2002). 